\title{
Finite $p$-supersoluble groups with some $E$-S-supplemented subgroups
}

\author{
Changwen Li $(*)$ - XiaOLONG YU $(* *)$ - NA TANG $(\ddagger)$
}

\begin{abstract}
Let $H$ be a subgroup of a group $G$ and $H_{e G}$ denote the subgroup of $H$ generated by all those subgroups of $H$ which are $S$-permutably embedded in $G$. $H$ is said to be $E$-S $S$-supplemented in $G$ if there exists a subnormal subgroup $T$ of $G$ such that $G=H T$ and $H \cap T \leq H_{e G}$. In this paper, we investigate the influence of some $E$-S -supplemented subgroups on the structure of finite groups. Some new characterizations of $p$-supersoluble groups are obtained.
\end{abstract}

Mathematics Subject Classification (2010). 20D10, 20 D20.

KEYwords. $S$-permutable, $E$-S-supplemented, $p$-supersoluble.

\section{Introduction}

Throughout this paper, all groups are finite. Most of the notation is standard and can be found in [4] and [17]. G always denotes a finite group and $p$ denotes a prime. $G$ is said to be $p$-nilpotent if it has a normal $p$-complement and $G$ is called a $p$-supersoluble group if its every $p$-chief factor is cyclic. It is

(*) Indirizzo dell'A.: School of Mathematics and Statistics, Jiangsu Normal University, Xuzhou, 221116, China.

E-mail: lcw2000@126.com

Supported by NNSF of China (Grant No. 11401264) and the Priority Academic Program Development of Jiangsu Higher Education Institutions.

(**) Indirizzo dell'A.: School of Mathematical Sciences, University of Science and Technology of China, Hefei 230026, China.

E-mail: yuxiaolong0710@sina.com

Supported by NNSF of China (Grant No. 11071229) and Specialized Research Fund for the Doctoral Program of Higher Education of China (Grant No. 20113402110036).

$(\ddagger)$ Indirizzo dell'A.: School of Mathematical Science, Huaiyin Normal University, Huaiyin, 223300, China.

E-mail: hytn999@126.com

Supported by Tianyuan foundation (Grant No. 11226285). 
easy to see that if $G$ is $p$-nilpotent then $G$ is also $p$-supersoluble. In the sequel, $\mathcal{U}_{p}$ will denote the class of all $p$-supersoluble groups and $G^{\mathcal{U}_{p}}$ will denote the $\mathcal{U}_{p}$-residual of $G$, i.e., the intersection of all normal subgroups $N$ of $G$ with $G / N \in \mathcal{U}_{p}$.

Recall that a subgroup $H$ of $G$ permutes with a subgroup $K$ of $G$ if $H K$ is a subgroup of $G$; $H$ is said to be $S$-permutable in $G$ if $H$ permutes with all Sylow subgroups of $G$. This property extends normality and permutability and was introduced by Kegel [10] in 1962. It turns out to be useful in establishing results concerning the group structure. On the other hand, the study of the generalizations of $S$-permutability is also one of the most fruitful research areas. For instance, Ballester-Bolinches and PedrazaAguilera [2] said that $H$ is $S$-permutably embedded in $G$, if for each prime $p$ dividing the order of $|H|$, a Sylow $p$-subgroup of $H$ is also a Sylow $p$-subgroup of some $S$-permutable subgroup of $G$. Skiba [20] called that $H$ is weakly $S$-permutable in $G$ if there is a subnormal subgroup $T$ of $G$ such that $G=H T$ and $H \cap T \leq H_{s G}$, where $H_{s G}$ is the subgroup of $H$ generated by all those subgroups of $H$ which are $S$-permutable in $G$. Using these generalized permutable subgroups, one has given a series of necessary and sufficient conditions for nilpotency, supersolubility, existence of Sylow towers and, more generally, for belonging to saturated formations containing some of these classes (see $[1,11,14,18,19])$. In order to unify above mentioned subgroups, Li, Zhang and Yi in [12] introduced the following concept:

DeFinition 1.1. Let $H$ be a subgroup of $G$ and $H_{e G}$ denote the subgroup of $H$ generated by all those subgroups of $H$ which are $S$-permutably embedded in $G$. If there is a subnormal subgroup $T$ of $G$ such that $G=H T$ and $H \cap T \leq H_{e G}$, then $H$ is called $E$-S - -supplemented in $G$.

In [12], the authors improved a nice result of Skiba which gives some conditions under which every $G$-chief factor of a normal subgroup $E$ of $G$ is cyclic. In present paper, we continue the work of [12] to study the influence of $E$-S-supplemented on the structure of finite groups. More precisely, we study $p$-supersolubility of finite groups under the assumption that some special subgroups are $E$-S $S$-supplemented.

\section{Preliminaries}

Lemma 2.1 ([12, Lemma 2.2]). Suppose that H is E-S-supplemented in G.

(1) If $H \leq L \leq G$, then $H$ is $E$-S-supplemented in $L$. 
(2) If $N \unlhd G$ and $N \leq H \leq G$, then $H / N$ is $E$-S-supplemented in $G / N$.

(3) If $H$ is a $\pi$-subgroup and $N$ is a normal $\pi^{\prime}$-subgroup of $G$, then $H N / N$ is $E$-S-supplemented in $G / N$.

Lemma 2.2 ([14, Lemma 2.3]). Suppose that $H$ is $S$-permutable in $G$, and let $P$ be a Sylow p-subgroup of $H$. If $H_{G}=1$, then $P$ is $S$-permutable in $G$.

Lemma 2.3 ([13, Lemma 2.6]). Assume that $L$ is a nontrivial normal subgroup of $G$ and $L \cap \Phi(G)=1$. Then the Fitting subgroup $F(L)$ of $L$ lies in $\operatorname{Soc}(G)$ and therefore $F(L)$ is the direct product of the minimal normal subgroups of $G$ contained in $F(L)$.

Lemma 2.4 ([3, Theorem 2.1.6]). If $G$ is p-supersoluble and $O_{p^{\prime}}(G)=1$, then the Sylow p-subgroup of $G$ is normal in $G$.

\section{Main Results}

THEOREM 3.1. Let $p$ be a fixed prime dividing the order of $G$ and $L$ a $p$ soluble normal subgroup of $G$ such that $G / L$ is p-supersoluble. If there exists a Sylow p-subgroup $P$ of $L$ such that every maximal subgroup of $P$ is $E$-S-supplemented in $G$, then $G$ is p-supersoluble.

Proof. Suppose that the theorem is false and let $G$ be a counterexample of minimal order. We will derive a contradiction in several steps.

(1) $G$ is $p$-soluble.

This follows directly from the $p$-solubility of $L$ and the $p$-supersolubility of $G / L$.

(2) $O_{p^{\prime}}(G)=1$.

If $T=O_{p^{\prime}}(G) \neq 1$, we consider $\bar{G}=G / T$. Clearly, $\bar{G} / \bar{L} \cong G / L T \cong$ $(G / L) /(T L / L)$ is $p$-supersoluble by the $p$-supersolubility of $G / L$, where $\bar{L}=L T / T$. Let $\overline{P_{1}}=P_{1} T / T$ be a maximal subgroup of $P T / T$. We may assume that $P_{1}$ is a maximal subgroup of $P$. Since $P_{1}$ is $E$-S $S$-supplemented in $G$, the subgroup $P_{1} T / T$ is $E$-S-supplemented in $G / T$ by Lemma 2.1(3). The minimal choice of $G$ yields that $\bar{G}$ is $p$-supersoluble, and so $G$ is also $p$ supersoluble, a contradiction.

(3) If $N$ is a minimal normal subgroup of $G$, then $N$ is an elementary abelian $p$-group. 
This follows from Steps (1) and (2).

(4) $G$ has a unique minimal normal subgroup $N$ contained in $L$ such that $G / N$ is $p$-supersoluble.

Let $N$ be a minimal normal subgroup of $G$ contained in $L$. Obviously, $N \leq P$ and $P / N$ is a Sylow $p$-subgroup of $L / N$. Let $P_{1} / N$ be a maximal subgroup of $P / N$. Then $P_{1}$ is a maximal subgroup of $P$. By hypothesis, $P_{1}$ is $E$-S-supplemented in $G$ and so $P_{1} / N$ is $E$-S $S$-supplemented in $G / N$ by Lemma 2.1(2). Since $(G / N) /(L / N) \cong G / L$ is $p$-supersoluble, $G / N$ satisfies all the hypotheses of our theorem. It follows that $G / N$ is $p$-supersoluble by the minimality of $G$. Noticing that the class of all $p$-supersoluble groups is a saturated formation, we have $N$ is the unique minimal normal subgroup of $G$ contained in $L$.

(5) $|N|>p$.

It is clear by Step (4).

(6) The final contradiction.

If $N$ is contained in all maximal subgroups of $G$, then $N \leq \Phi(G)$ and so $G$ is $p$-supersoluble. This contradiction shows that there exists a maximal subgroup $M$ of $G$ such that $G=N M$ and $N \cap M=1$. Let $G_{p}$ be a Sylow $p$ subgroup of $G$ containing $P$. Then $G_{p}=N\left(G_{p} \cap M\right)$ and $G_{p} \cap M<G_{p}$. Take a maximal subgroup $G_{1}$ of $G_{p}$ containing $G_{p} \cap M$ and set $P_{1}=G_{1} \cap P$. Then $\left|P: P_{1}\right|=\left|P: G_{1} \cap P\right|=\left|P G_{1}: G_{1}\right|=\left|G_{p}: G_{1}\right|=p$ and so $P_{1}$ is a maximal subgroup of $P$. First, we have $N \nsubseteq P_{1}$. If not, $P=P \cap G_{p}=$ $P \cap N G_{1}=N\left(G_{1} \cap P\right)=N P_{1}=P_{1}$, a contradiction. Secondly, we have $N \cap P_{1} \neq 1$. If not, $\left|N: P_{1} \cap N\right|=\left|N P_{1}: P_{1}\right|=\left|P: P_{1}\right|=p$ and so $P_{1} \cap N$ is a maximal of $N$. Therefore $|N|=p$, which contradicts Step (5).

By hypothesis, $P_{1}$ is $E$-S $S$-supplemented in $G$. Then there is a subnormal subgroup $T$ of $G$ such that $G=P_{1} T$ and $P_{1} \cap T \leq\left(P_{1}\right)_{e G}$. Since $|G: T|$ is a power of $p$ and $T \triangleleft \triangleleft G, O^{p}(G) \leq T$. We know $G / O^{p}(G)$ is a $p$-subgroup, so $G / O^{p}(G)$ is $p$-supersoluble and $G /\left(N \cap O^{p}(G)\right) \lesssim G / N \times G / O^{p}(G)$ is $p$-supersoluble. Then $N \cap O^{p}(G) \neq 1$. Since $N$ is the minimal subgroup of $G$, $N \cap O^{p}(G)=N$ and so $N \leq O^{p}(G)$. It follows that $N \cap P_{1}=N \cap\left(P_{1}\right)_{e}$. Obviously, $\left(P_{1}\right)_{e G} \neq 1$. Let $U_{1}, U_{2}, \ldots, U_{s}$ be all the nontrivial subgroups of $P_{1}$ which are $S$-permutably embedded in $G$. For every $i \in\{1,2, \ldots, s\}$, then there is an $S$-permutable subgroup $K_{i}$ of $G$ such that $U_{i}$ is a Sylow $p$ subgroup of $K_{i}$. Suppose that for some $i \in\{1,2, \ldots, s\}$, we have $\left(K_{i}\right)_{G} \neq 1$. Then we can take a minimal normal subgroup $D$ of $G$ such that $D \leq\left(K_{i}\right)_{G}$. In view of Step (3), $D$ is a $p$-group. Then $D \leq\left(P_{1}\right)_{e G} \leq P_{1} \leq L$. By virtue of Step (4), $D=N$. Consequently, $N \leq P_{1}$. This contradiction shows 
that for all $i \in\{1,2, \ldots, s\}$, we have $\left(K_{i}\right)_{G}=1$. By Lemma 2.2, $U_{i}(i \in$ $\{1,2, \ldots, s\})$ are $S$-permutable in $G$. It follows that $\left(P_{1}\right)_{e G}$ is $S$-permutable in $G$. Let $G_{q}$ be any Sylow $q$-subgroup of $G$ with $p \neq q$. Then $\left(P_{1}\right)_{e G} G_{q}=G_{q}\left(P_{1}\right)_{e G}$. Since $N \cap P_{1}=N \cap\left(P_{1}\right)_{e G}=N \cap\left(P_{1}\right)_{e} G_{q}$, we have $N \cap P_{1}$ is normalized by $G_{q}$. Since $P=G_{p} \cap L$, we have $P \unlhd G_{p}$ and so $P_{1} \unlhd G_{p}$. It follows that $N \cap P_{1}$ is normalized by $G_{p}$. Therefore, $N \cap P_{1}$ is normal in $G$. The minimality of $N$ implies that $N \cap P_{1}=1$ or $N \subseteq P_{1}$, a contradiction.

We can choose $L$ to get some results of special interest. For example, if we choose $L=G$ or $L=G^{\mathcal{U}_{p}}$, then we obtain the following criteria for $p$ supersolubility of groups.

Corollary 3.2. Let P be a Sylow p-subgroup of a p-soluble $G$, where $p$ is a fixed prime divisor of $|G|$. If all maximal subgroups of $P$ are $E-S$ supplemented in $G$, then $G$ is p-supersoluble.

Corollary 3.3. A p-soluble $G$ is p-supersoluble if and only if all maximal subgroups of any Sylow p-subgroup of $G^{\mathcal{U}_{p}}$ are E-S-supplemented in $G$.

In the following, we shall denote by $F_{p}(G)$ the $p$-Fitting subgroup of $G$. In fact, $F_{p}(G)=O_{p^{\prime} p}(G)$.

THEOREM 3.4. Let $p$ be a fixed prime dividing the order of $G$ and $L$ a $p$ soluble normal subgroup of $G$ such that $G / L$ is $p$-supersoluble. If all maximal subgroups of $F_{p}(L)$ containing $O_{p^{\prime}}(L)$ are $E$-S-supplemented in $G$, then $G$ is p-supersoluble.

Proof. Suppose that the theorem is false and let $G$ be a counterexample of minimal order. We will derive a contradiction in several steps.

(1) $O_{p^{\prime}}(L)=1$.

If $O_{p^{\prime}}(L) \neq 1$, we consider the factor group $G / O_{p^{\prime}}(L)$. First,

$$
\left(G / O_{p^{\prime}}(L)\right) /\left(L / O_{p^{\prime}}(L)\right) \cong G / L
$$

is $p$-supersoluble. Now $O_{p^{\prime}}\left(L / O_{p^{\prime}}(L)\right)=1$ and $F_{p}\left(L / O_{p^{\prime}}(L)\right)=F_{p}(L) / O_{p^{\prime}}(L)$. Let $M / O_{p^{\prime}}(L)$ be a maximal subgroup of $F_{p}\left(L / O_{p^{\prime}}(L)\right)$. Then $M$ is a maximal subgroup of $F_{p}(L)$ containing $O_{p^{\prime}}(L)$. Since $M$ is $E$-S $S$-supplemented in $G$, in view of Lemma 2.1(2), $M / O_{p^{\prime}}(L)$ is $E$-S-supplemented in $G / O_{p^{\prime}}(L)$. Thus 
$G / O_{p^{\prime}}(L)$ satisfies the hypotheses of the theorem. The minimality of $G$ implies that $G / O_{p^{\prime}}(L)$ is $p$-supersoluble and so $G$ is $p$-supersoluble, contrary to the choice of $G$.

(2) $L \cap \Phi(G)=1$.

Write $H=L \cap \Phi(G)$. If $H \neq 1$, we consider the factor group $G / H$. By virtue of [9, III, 3.5], we have $F(L / H)=F(L) / H$ and so $F(L / H)=$ $O_{p}(L) / H$. On the other hand, writing $K / H=O_{p^{\prime}}(L / H)$ and letting $S$ be a Hall $p^{\prime}$-subgroup of $K$ we have $K=S H$, and by the Frattini argument $G=K N_{G}(S)=H N_{G}(S)=N_{G}(S)$ and $S \triangleleft G$. Therefore $S=1$ and $O_{p^{\prime}}(L / H)=1$. This implies that $F_{p}(L / H)=O_{p}(L / H)=O_{p}(L) / H=$ $F_{p}(L) / H$. If $P_{1} / H$ is a maximal subgroup of $F_{p}(L / H)$, then $P_{1}$ is maximal in $F_{p}(L)$ and, by our hypothesis, it is an $E$-S $S$-supplemented subgroup of $G$. Hence $P_{1} / H$ is $E$-S-supplemented in $G / H$ by Lemma 2.1(2). Now the minimality of $G$ implies that $G / H$ is $p$-supersoluble and then so is $G$, which contradicts the choice of $G$.

(3) The final contradiction.

Since $L$ is $p$-soluble and $O_{p^{\prime}}(L)=1$, we have $C_{L}\left(O_{p}(L)\right) \leq O_{p}(L)$ by [5, Theorem 6.3.2]. Now $\Phi(L)=1$ implies that $F(L)=O_{p}(L)$ is a nontrivial elementary abelian $p$-group by [9, III, 4.5]. Thus $C_{L}(F(L))=F(L)$. By hypothesis, all maximal subgroups of $F(L)$ are $E$-S -supplemented in $G$. Applying [12, Theorem 1.4], all $G$-chief factors of $F(L)$ are cyclic. In view of Lemma 2.3, we have $F(L)=N_{1} \times N_{2} \times \cdots \times N_{r}$, where $N_{i}$ is a minimal normal of $G$ with order $p$. Since for each $i$ the factor group $G / C_{G}\left(N_{i}\right)$ is isomorphic to some subgroup of $\operatorname{Aut}\left(N_{i}\right)$, we have $G / C_{G}\left(N_{i}\right)$ is cyclic. Consequently, $G / C_{G}\left(N_{i}\right)$ is $p$-supersoluble. From the $p$-supersolubility of $G / L$, it follows that $G /\left(L \cap C_{G}\left(N_{i}\right)\right)=G / C_{L}\left(N_{i}\right)$ is $p$-supersoluble. Therefore $G / \bigcap_{i=1}^{r} C_{L}\left(N_{i}\right)$ is $p$-supersoluble. In fact, what we really have is $G / F(L)$ is $p$-supersoluble because

$$
\bigcap_{i=1}^{r} C_{L}\left(N_{i}\right)=C_{L}(F(L))=F(L) .
$$

However all $G$-chief factors of $F(L)$ are cyclic of order $p$ and hence $G$ is $p$ supersoluble, a contradiction.

COROLLARY 3.5. Let $G$ be a p-soluble group, where $p$ is a fixed prime divisor of $|G|$. Then $G$ is p-supersoluble if and only if all maximal subgroups of $F_{p}\left(G^{\mathcal{U}_{p}}\right)$ containing $O_{p^{\prime}}\left(G^{\mathcal{U}_{p}}\right)$ are $E$-S-supplemented in $G$. 
THEOREM 3.6. Let $p$ be a prime dividing the order of $G$ and $P a$ Sylow p-subgroup of $G$. If $N_{G}(P)$ is p-nilpotent and all maximal subgroups of $P$ are $E$-S-supplemented in $G$, then $G$ is p-nilpotent. In particular, $G$ is p-supersoluble.

Proof. It is easy to see that the theorem holds when $p=2$ by $[12$, Theorem 5.1], so it suffices to prove the theorem for the case of odd prime.

Suppose that the theorem is false and let $G$ be a counterexample of minimal order.

(1) If $H$ is a proper subgroup of $G$ with $P \leq H<G$, then $H$ is $p$-nilpotent.

It is easy to see that $N_{H}(P) \leq N_{G}(P)$ and hence $N_{H}(P)$ is $p$-nilpotent. By Lemma 2.1(1), all maximal subgroups of $P$ are $E$-S-supplemented in $H$. Hence $H$ satisfies the hypothesis of our theorem. The minimal choice of $G$ implies that $H$ is $p$-nilpotent.

(2) $O_{p^{\prime}}(G)=1$.

If $O_{p^{\prime}}(G) \neq 1$, we consider $G / O_{p^{\prime}}(G)$. Obviously, $P O_{p^{\prime}}(G) / O_{p^{\prime}}(G)$ is a Sylow $p$-subgroup of $G / O_{p^{\prime}}(G)$. In view of Lemma 2.1(3), it is easy to see that all maximal subgroups of $P O_{p^{\prime}}(G) / O_{p^{\prime}}(G)$ are $E$-S-supplemented in $G / O_{p^{\prime}}(G)$. Since

$$
N_{G / O_{p^{\prime}}(G)}\left(P O_{p^{\prime}}(G) / O_{p^{\prime}}(G)\right)=N_{G}(P) O_{p^{\prime}}(G) / O_{p^{\prime}}(G)
$$

is $p$-nilpotent, $G / O_{p^{\prime}}(G)$ satisfies the hypothesis of our theorem. The minimal choice of $G$ yields that $G / O_{p^{\prime}}(G)$ is $p$-nilpotent and so $G$ is $p$-nilpotent, a contradiction.

(3) $O_{p}(G) \neq 1$.

Let $J(P)$ be the Thompson subgroup of $P$. Then $N_{G}(P) \leq$ $N_{G}(Z(J(P))) \leq G$. If $N_{G}(Z(J(P)))<G$, then, in view of Step (1), $N_{G}(Z(J(P)))$ is $p$-nilpotent and so $G$ is $p$-nilpotent by [5, Theorem 8.3.1], a contradiction. Hence $N_{G}(Z(J(P)))=G$, which shows that $Z(J(P))$ is a normal $p$-subgroup of $G$ and $1<O_{p}(G)<P$.

(4) $G$ is $p$-soluble.

It is easy to see that the factor group $G / O_{p}(G)$ satisfies the hypothesis of our theorem. Now, by the minimality of $G$, we see that $G / O_{p}(G)$ is $p$ nilpotent. Consequently, $G / O_{p}(G)$ is $p$-soluble and so $G$ is $p$-soluble.

(5) The final contradiction.

Applying Corollary 3.2, $G$ is $p$-supersoluble. In view of Lemma 2.4, $P$ is normal in $G$. Therefore, $G=N_{G}(P)$ is $p$-nilpotent, a contradiction. 
CoRollary 3.7. Let $p$ be a prime dividing the order of $G$ and $L a$ normal subgroup of $G$ such that $G / L$ is p-nilpotent. Suppose that there exists a Sylow p-subgroup $P$ of $L$ such that all maximal subgroups of $P$ are $E$-S-supplemented in $G$ and $N_{G}(P)$ is p-nilpotent. Then $G$ is p-nilpotent.

Proof. It is obvious that $N_{L}(P)$ is $p$-nilpotent and all maximal subgroups of $P$ are $E$-S-supplemented in $L$. Applying Theorem 3.6, $L$ is $p$-nilpotent. Now we have $L_{p^{\prime}}$ is the normal Hall $p^{\prime}$-subgroup of $L$. Furthermore, $L_{p^{\prime}}$ is a normal subgroup of $G$. If $L_{p^{\prime}} \neq 1$, we consider the factor group $G / L_{p^{\prime}}$. First, $\left(G / L_{p^{\prime}}\right) /\left(L / L_{p^{\prime}}\right) \cong G / L$ is $p$-nilpotent and all maximal subgroups of $P L_{p^{\prime}} / L_{p^{\prime}}$ are $E$-S-supplemented in $G / L_{p^{\prime}}$ by Lemma 2.1(3). Secondly, $N_{G / L_{p^{\prime}}}\left(P L_{p^{\prime}} / L_{p^{\prime}}\right)=N_{G}(P) L_{p^{\prime}} / L_{p^{\prime}}$ is $p$-nilpotent. Hence $G / L_{p^{\prime}}$ satisfies the hypothesis of our corollary. By induction, $G / L_{p^{\prime}}$ is $p$-nilpotent and so $G$ is $p$-nilpotent, as desired. Hence we may assume $L_{p^{\prime}}=1$, i.e., $L=P$. By hypothesis, $N_{G}(P)=G$ is $p$-nilpotent.

THEOREM 3.8. Let $L$ be a normal subgroup of $G$ such that $G / L$ is $p$ supersoluble, where $p$ is a prime divisor of $|L|$ with $(p-1,|L|)=1$. Suppose that for a Sylow p-subgroup $P$ of L, there exists a subgroup $D$ of $P$ such that $1<|D|<|P|$ and every subgroup $H$ of $P$ with order $|H|=|D|$ and every cyclic subgroup of $P$ with order 4 (if $|D|=2$ and $P$ is a non-abelian 2-group) is E-S-supplemented in $G$. Then $G$ is p-supersoluble.

Proof. Suppose that this theorem is false and consider a counterexample $(G, L)$ for which $|G||L|$ is minimal.

(1) $L$ is $p$-nilpotent.

By Lemma 2.1, it is easy to see that every subgroup $H$ of $P$ with order $|H|=|D|$ and every cyclic subgroup of $P$ with order 4 (if $|D|=2$ and $P$ is a non-abelian 2-group) are $E$-S-supplemented in $L$. Applying [12, Theorem 1.5], $L$ is $p$-nilpotent.

(2) $P=L$.

From Step 1, we know $O_{p^{\prime}}(L)$ is the normal Hall $p^{\prime}$-subgroup of $L$. Assume that $O_{p^{\prime}}(L) \neq 1$. In view of Lemma 2.1, the hypothesis holds for $\left(G / O_{p^{\prime}}(L), L / O_{p^{\prime}}(L)\right)$. Hence, by the minimal choice of $(G, L)$, the theorem is true for $\left(G / O_{p^{\prime}}(L), L / O_{p^{\prime}}(L)\right)$ and so $G / O_{p^{\prime}}(L)$ is $p$-supersoluble. Consequently, $G$ is $p$-supersoluble. This contradiction shows that $O_{p^{\prime}}(L)=1$. Hence $L$ is a normal $p$-subgroup of $G$.

(3) The final contradiction. 
Applying [12, Theorem 1.4], all $G$-chief factors of $L$ are cyclic. From the $p$-supersolubility of $G / L$, we have $G$ is $p$-supersoluble.

CoRollary 3.9. Let $p$ be a prime divisor of $\left|G^{\mathcal{U}_{p}}\right|$ with $\left(p-1,\left|G^{\mathcal{U}_{p}}\right|\right)=1$. Suppose that for a Sylow p-subgroup $P$ of $G^{\mathcal{U}_{p}}$, there exists a subgroup $D$ of $P$ such that $1<|D|<|P|$ and every subgroup $H$ of $P$ with order $|H|=|D|$ and every cyclic subgroup of $P$ with order 4 (if $|D|=2$ and $P$ is a nonabelian 2-group) is E-S-supplemented in $G$. Then $G$ is p-supersoluble.

Corollary 3.10. Let $p$ be the smallest prime divisor of $|G|$. Suppose that for a Sylow p-subgroup $P$ of $G^{\mathcal{U}_{p}}$, there exists a subgroup $D$ of $P$ such that $1<|D|<|P|$ and every subgroup $H$ of $P$ with order $|H|=|D|$ and every cyclic subgroup of $P$ with order 4 (if $|D|=2$ and $P$ is a non-abelian 2-group) is E-S-supplemented in $G$. Then $G$ is p-nilpotent.

Proof. By virtue of Corollary 3.9, $G$ is $p$-supersoluble. Since $p$ is the smallest prime divisor of $|G|$, we have $G$ is $p$-nilpotent by [11, Lemma 2.8].

\section{Some Applications}

It is clear that all the subgroups, whether they are $c$-normal subgroups [21], $c^{*}$-normal subgroups [22], $S$-permutably embedded subgroups or weakly $S$-permutable subgroups, are all $E$-S-supplemented subgroups. Hence the following results are all special cases of our Theorems.

Corollary 4.1 ([22, Theorem 3.7]). Let $p$ be a prime, G a p-soluble group and $H$ a normal subgroup of $G$ such that $G / H$ is p-supersoluble. If all maximal subgroups of $F_{p}(H)$ containing $O_{p^{\prime}}(H)$ are $c^{*}$-normal in $G$, then $G$ is p-supersoluble.

Corollary 4.2 ([22, Theorem 3.5]). Let $p$ be a prime, G a p-soluble group and $H$ a normal subgroup of $G$ such that $G / H$ is p-supersoluble. If there exists a Sylow p-subgroup $P$ of $H$ such that every maximal subgroup of $P$ is $c^{*}$-normal in $G$, then $G$ is $p$-supersoluble.

Corollary 4.3 ([16, Theorem 3.1]). Let $p$ be a prime, G a p-soluble group and $H$ a normal subgroup of $G$ such that $G / H$ is p-supersoluble. If there exists a Sylow p-subgroup $P$ of $H$ such that every maximal subgroup of $P$ is c-normal in $G$, then $G$ is p-supersoluble. 
Corollary 4.4 ([8, Theorem 3.10]). Let $p$ be a prime, $G$ a p-soluble group and $H$ a normal subgroup of $G$ such that $G / H$ is p-supersoluble. If there exists a Sylow p-subgroup $P$ of $H$ such that every maximal subgroup of $P$ is $S$-permutably embedded in $G$, then $G$ is p-supersoluble.

COROLlary 4.5 ([15, Theorem 3.3]). Let $p$ be a prime and $G$ a $p$ soluble group. If there exists a Sylow p-subgroup $P$ of $G$ such that every maximal subgroup of $P$ is weakly $S$-permutable in $G$, then $G$ is $p$ supersoluble.

Corollary 4.6 ([6, Theorem 3.1]). Let $p$ be an odd prime dividing the order of $G$ and $P$ a Sylow p-subgroup of $G$. If $N_{G}(P)$ is p-nilpotent and every maximal subgroup of $P$ is c-normal in $G$, then $G$ is $p$ nilpotent.

Corollary 4.7 ([14, Theorem 3.2]). Let $p$ be a prime dividing the order of $G$ and $P$ a Sylow p-subgroup of $G$. If $N_{G}(P)$ is p-nilpotent and every maximal subgroup of $P$ is $S$-permutably embedded in $G$, then $G$ is p-nilpotent.

\section{Some Remarks}

REMaRK 5.1. The hypothesis that $L$ is $p$-soluble in Theorems 3.1 and 3.4 cannot be removed. Consider for example the group $G=A_{5}$, the alternating group of degree 5 . Clearly 1 is the maximal subgroup of any Sylow 5-subgroup of $G$ and $F_{5}(G)=1$. However, $G$ is not 5 -supersoluble.

Remark 5.2. In proving our Theorem 3.6, the assumption that $N_{G}(P)$ is $p$-nilpotent is essential. To illustrate the situation, we may also consider $G=A_{5}$ and $p=5$.

REMARK 5.3. From [7] or [11], we know if there is a subgroup $T$ of $G$ such that $G=H T$ and $H \cap T \leq H_{e G}$, then $H$ is called $S E$-supplemented or $E$-supplemented in $G$. Obviously, the set of all $S E$-supplemented subgroups of a group is wider than the set of all its $E-S$-supplemented subgroups. However, the following example shows that we cannot generalize our Theorems 3.1 and 3.6 using $S E$-supplemented subgroups: Let $A=Z_{3} \times Z_{2} \cong S_{3}$, where $Z_{3}$ is a cyclic subgroup of order $3, Z_{2}$ is a cyclic 
subgroup of order 2 and $S_{3}$ is the symmetric group of degree 3. Let $B=A<Z_{3}$, the regular wreath product of $A$ by $Z_{3}$. Put $G=O^{2}(B)=$ $\langle x \mid \circ(x)=3\rangle$. Then $G \cong\left(Z_{3} \times Z_{3} \times Z_{3}\right) \times A_{4}$ and $G$ is soluble, where $A_{4}$ is the alternating group of degree 4 . Let $P$ be a Sylow 3-subgroup of $G$. Then $N_{G}(P)=P$ is 3-nilpotent. It is easy to see that every maximal subgroup of $P$ is $S E$-supplemented in $G$. But $G$ is not 3 -supersoluble.

Acknowledgments. The authors would like to thank the referee who read the manuscript carefully and contributed a lot of valuable suggestions and useful comments.

\section{REFERENCES}

[1] M. AsaAd and A. A. Heliel, On S-quasinormally embedded subgroups of finite groups, J. Pure Appl. Algebra, 165 (2001), pp. 129-135.

[2] A. Ballester-Bolinches and M. C. Pedraza-Aguilera, Sufficient conditions for supersolubility of finite groups, J. Pure Appl. Algebra, 127 (1998), pp. $113-118$.

[3] A. Ballester-Bolinches, R. Esteban-Romero and M. Asaad, Products of finite groups, Walter de Gruyter, Berlin, 2010.

[4] K. Doerk and T. Hawkes, Finite solvable Groups, Walter de Gruyter, BerlinNew York, 1992.

[5] D. Gorenstein, Finite Groups, Harper and Row, New York, 1968.

[6] X. Guo and K. P. SHum, On c-normal maximal and minimal subgroups of Sylow p-subgroups of finite groups, Arch. Math., 80 (2003), pp. 561-569.

[7] W. Guo, A. N. Skiba and N. YANG, SE-supplemented subgroups of finite groups, Rend. Sem. Mat. Univ. Padova, 129 (2013), pp. 245-263.

[8] A. A. Heliel and S. M. Alharbia, The influence of certain permutable subgroups on the structure of finite groups, Int. J. Algebra, 4 (2010), pp. 1209-1218.

[9] B. Huppert, Endliche Gruppen I, Springer-Verlag, Berlin-New York, 1967.

[10] O. H. KEGEL, Sylow Gruppen und subnormalteiler endlicher Gruppen, Math. Z., 78 (1962), pp. 205-221.

[11] C. LI, X. ZHANG and Y. WANG, On E-supplemented subgroups and the structure of finite groups, J. Algebra Appl., 12 (2013), 1350019.

[12] C. LI, X. ZHANG and X. YI, On E-S-supplemented subgroups of finite groups, Colloq. Math., 131 (2013), pp. 41-51.

[13] Y. LI, Y. WANG and H. WEI, The influence of $\pi$-quasinormality of some subgroups of a finite group, Arch. Math., 81 (2003), pp. 245-252.

[14] Y. LI, Y. WANG and H. WEI, On p-nilpotency of finite groups with some subgroups $\pi$-quasinormally embedded, Acta Math. Hungar., 108 (2005), pp. 283-298.

[15] L. Miao, On weakly S-permutable subgroups, Bull. Braz. Math. Soc, New Series, 41 (2010), pp. 223-235. 
[16] M. Ramadan, M. Ezzat-Mohamed and A. A. Heliel, On c-normality of certain subgroups of prime power order of finite groups, Arch. Math., 85 (2005), pp. 203-210.

[17] D. J. S. Robinson, A Course in Theory of Group, Springer-Verlag, 1982.

[18] L. A. Shemetkov and A. N. SkiBa, On the X $\mathcal{X}$-hypercentre of finite groups, J. Algebra, 322 (2009), pp. 2106-2117.

[19] A. N. SkIBA, Cyclicity conditions for G-chief factors of normal subgroups of a group G, Siberian Math. J., 52 (2011), pp. 127-130.

[20] A. N. SkiBA, On weakly S-permutable subgroups of finite groups, J. Algebra, 315 (2007), pp. 192-209.

[21] Y. WANG, c-Normality of groups and its properties, J. Algebra, 180 (1996), pp. 954-965.

[22] H. WeI and Y. WANG, On $c^{*}$-normality and its properties, J. Group Theory, 10 (2007), pp. 211-223.

Manoscritto pervenuto in redazione il 12 luglio 2013. 